\title{
Valores de impacto no corpo do judoca ao ser projetado pela técnica Ippon-Seoi-Nage
}

\author{
T. Piucco, S.G. Santos
}

\begin{abstract}
Este estudo exploratório objetivou analisar as magnitudes e o tempo de duração dos impactos gerados em diferentes regióes corpo do judoca projetado pela técnica Ippon-SeoiNage. Participaram dois judocas faixas pretas, sendo tori (judoca que projeta) com $75 \mathrm{~kg}$ e 19 anos de prática, e o uke (judoca que cai) com $72 \mathrm{~kg}$ e 15 anos de prática. Foi utilizado um acelerômetro triaxial 4321 da Brüel \& Kjaer fixado no punho, quadril e tornozelo do uke. Os dados foram coletados em ambiente laboratorial, sendo realizadas 10 quedas para cada articulação investigada, totalizando 30 quedas. Os maiores valores de impacto ocorreram no punho no eixo vertical $(351.95 \mathrm{~g})$ e os menores valores no quadril no eixo antero-posterior (5.18 g); os tempos de duração dos impactos foram maiores no quadril no eixo vertical ( $.58 \mathrm{~s}$ ) não havendo diferença entre o punho e o tornozelo. Considerando os altos valores de impacto encontrados, bem como o elevado número de repetição de quedas por treino no judô, sugere-se que sejam revistas as metodologias de práticas e o tipo de tatame utilizado, objetivando minimizar os possíveis efeitos deletérios dos impactos ao organismo dos judocas em longo prazo.
\end{abstract}

Palavras-chave: judô, impacto, ukemi

\section{Magnitude and duration of the impact generated on the athletes' body during training in Ippon-Seoi-Nage judo technique}

This exploratory study aimed to analyse the magnitude and duration of the impact generated on the athletes' body during training in Ippon-Seoi-Nage judo technique. Two black belt judo athletes were studied, the tori (the thrower) weighting $75 \mathrm{~kg}$ and having 19 years of practice, and the $u k e$ (the thrown) weighting $72 \mathrm{~kg}$ and having 15 years of practice. A Bruel \& Kjaer 4321 Accelerometer Triaxial, fastened to the fist, hip and ankle of the uke, was used. Data were collected in the laboratory. Ten falls were performed for each of the three articulations investigated. The largest impact values were observed for the fist in the vertical axis (351.95 g) and the smallest values were for the hip anterior posterior axis $(5.18 \mathrm{~g})$. Impact duration times were greater in the hip vertical axis $(.58 \mathrm{~s})$ with no differences for the impact on fist and ankle. Considering these high impact values as well as the high frequency of falls during judo training, it is suggested a reevaluation of this training pattern and the sort of tatami adopted, in order to decrease the resulting harmful effects over the time.

Keywords: judo, impact, ukemi

Tatiane Piucco. Universidade Federal de Santa Catarina, Pós-Graduação em Educação Física, Centro de Desportos, Laboratório de Biomecânica, Florianópolis, SC - Brasil

Saray Giovana dos Santos. Universidade Federal de Santa Catarina, Departamento de Educação Física, Centro de Desportos, Laboratório de Biomecânica, Florianópolis, SC - Brasil

Endereço para correspondência: Tatiane Piucco, Rua Capitão Romualdo de Barros, 694, AP 301C, Bairro Carvoeira, Florianópolis SC, CEP: 88040-600 - Brasil

E-mail: tatianepiucco@yahoo.com.br 
Muito embora os impactos mecânicos nos esportes sejam frequentes, poucos estudos investigam as magnitudes dos impactos geradas em esportes cujos fundamentos exigem quedas e/ou aterrissagens, a exemplo do judô e do voleibol, bem como as consequências destes impactos ao organismo do atleta. Este quadro pode ser justificado pela complexidade e dificuldades de se mensurar e verificar e os efeitos das vibrações mecânicas ao organismo, conforme afirma Griffin (1990).

Em virtude da complexidade dos fatores que determinam as respostas do homem a vibrações, foram estabelecidas normas para facilitar a avaliação e comparação dos dados e determinar os níveis de aceitação de exposição do corpo todo às vibrações (Santos, 2003). Referente a vibrações mecânicas do tipo periódicas, comuns em veículos, construções e aquelas provindas de equipamentos e nas proximidades de máquinas trabalhando, existem normas (ISO 2631,1985; ISO 5349,1986; BS 6841,1987) que limitam o tempo de exposição do corpo humano às mesmas. Porém, estas normas não são consideradas aplicáveis a exposições as vibrações do tipo transitórias, que contenham impactos mecânicos (choques) repetitivos, característicos dos impactos desportivos, e nem providencia meios para relacionar esses impactos com os efeitos para a saúde.

O único critério que se pode tomar como referência (entendendo a duração do pulso como o tempo total de impacto) para impactos esportivos é o citado por Macaulay (1987), o qual é plotado graficamente para três regiões, sendo uma região de curta duração para tempos de .001 a $.01 \mathrm{~s}$, uma região intermediária e uma de longa duração para tempos maiores que $.1 \mathrm{~s}$, com limite de impacto de 200 g. Este critério postula que quanto menores as durações dos eventos maiores acelerações o organismo suporta, sem ser acometidos de lesões. No entanto, nele não é citado o número de eventos que o organismo pode suportar por um determinado período de tempo assim como o intervalo entre as repetições dos mesmos.

Mesmo sem critérios específicos, estudos como os de Voloshin (2004), Lafortune, Henning e Valiant (1995), Derrick e Mercer (2003), entre outros, apontam a repetição dos movimentos esportivos como os principais mecanismos de lesões desportivas. Os valores de impacto podem chegar a $53.13 \pm$ $14.26 \mathrm{~g}$ aterrissagens de saltos do basquetebol, na articulação do tornozelo (Piucco, Santos, Pacheco, Souza, \& Piucco, 2007), a $334.88 \pm 64.66 \mathrm{~g}$ nos chutes de artes marciais como o taekwondo (Esteves, Nascimento, Moreira, $\&$ Reis, 2007); e, a $284 \pm 34$ g no tornozelo (Santos, 2003) e 351.95 g no punho (Piucco, 2007) nas quedas do judô. Alguns estudos têm investigado os impactos durante a rebatida no tênis, no cabo da raquete (Hatze, 1992) e no punho e no cotovelo dos atletas (Hennig, Rosenbaum, \& Milani, 1992). Outros identificaram as vibrações transmitidas a ciclistas com diferentes tipos de suspensão de bicicletas (Levy \& Smith, 2005; Orendurff, 1997), e aquelas transmitidas ao corpo de corredores (Boyer \& Nig, 2004; Jarrah, Qas- 
sem, Othman, \& Gdeisat, 1997; Gottschall \& Kram, 2005).

Nas artes marciais, a relação das lesões esportivas é bastante elevada, ficando em torno de 22.3 : 1000 (Leavitt, 2003). No judô, em atletas adultos do sexo masculino, a taxa de acometimento de lesões varia entre 25.2 e 122.6 : 1000 atletas expostos, e nas mulheres entre 34.3 e $130.6: 1000$ (Barrault, Achou, \& Sorel, 1983; James \& Pieter, 2003; Pieter, Talbot, Pinlac, $\&$ Bercades, 2001).

Muito embora os ukemi no judô tenham sido idealizados para amenizar os efeitos das quedas, dissipando no tatame no momento do impacto a energia cinética empreendida no corpo prevenindo assim os efeitos indesejáveis das vibrações, as lesões sofridas pelos judocas na sua maioria são resultantes de ukemi mal realizados (Santos \& Melo, 2003; Santos \& Shigunov, 2001). De acordo com Barsottini, Guimarães e Morais (2006), os golpes mais frequentes que ocasionam lesões no judô são o Ippon-Seoi-Nage, com 23\%, o Tai-Otoshi com $22 \%$ e o Uchi-Mata com $9 \%$, sendo estas técnicas mais utilizadas pelos judocas da categoria peso leve em competições. Além disso, podemos considerar que o grande número de quedas realizadas por judocas amadores, que fica em torno de 74 quedas por treino (Santos \& Melo, 2001), como um fator que pode contribuir para o aumento no acometimento de lesões.

Uma das preocupações referentes aos impactos sofridos na região do quadril é o acometimento de danos nos órgãos internos, situados na região do abdômen e quadril, os quais possuem maior sensibilidade em faixas de frequência baixas de 4 a $8 \mathrm{~Hz}$ (Harris \& Piersol, 2002; Ishitake, Kano, Miyazaki, Ando, Tsutsumi, \& Matoba, 1998). Segundo Sterkowicz (1999), os ataques inesperados no judô podem provocar danos corporais principalmente durante a projeção e a queda na região posterior do sacro.

O judô é uma das modalidades esportivas que apresenta o maior índice de adeptos mundialmente, principalmente entre indivíduos púberes e prépúberes (Fraga, 2002), e também é um dos esportes que mais apresenta lesões, muitas delas geradas durante as quedas. Por isso, este estudo se justifica por trazer dados importantes para orientar tanto na iniciação quanto para o treinamento do judô, considerando-se a importância do ensino e da execução correta dos ukemi de uma forma consciente e adequada à técnica do golpe realizado, bem como do número $\mathrm{e}$ intervalo de recuperação ideal entre as quedas. Esta pesquisa também disponibiliza dados que contribuirão para a realização de outros estudos afins, dedicados a construção de um banco de dados para futura determinação de limites de segurança para os impactos esportivos, visto que a interpretação estatística de modelos biomecânicos depende, em primeiro lugar, da expansão dos parâmetros fisiológicos e biomecânicos do movimento em questão.

Deste modo, tendo em vista a importância de se investigar variáveis que possam estar propiciando malefícios ao organismo dos atletas, é que para este estudo levantaram-se as seguintes questões a serem investiga- 
das: quais as magnitudes dos impactos que um judoca é submetido ao ser projetado pela técnica Ippon-Seoi-Nage? Será que os níveis vibratórios advindos desta queda estão em níveis geradores de lesões? Para responder as questões do estudo, objetivou-se investigar as características dos impactos em diferentes regiões corporais do judoca durante a queda proveniente da projeção da técnica Ippon-Seoi-Nage. Mais especificamente objetivou-se identificar as magnitudes e os tempos dos impactos gerados no punho, quadril e tornozelo no momento da queda; comparar as magnitudes e os tempos dos impactos entre os eixos de movimento (láterolateral, ântero-posterior e vertical) e entre as articulações corporais (punho, quadril e tornozelo).

\section{MÉTODO}

Participaram desse estudo do tipo exploratório dois judocas, escolhidos intencionalmente, sendo ambos com biótipo, graduação judoística (faixa preta) e nível técnico similares, competidores da categoria de peso leve (66 a $73 \mathrm{~kg}$ ) e meio médio (73 a $81 \mathrm{~kg}$ ). O tori (judoca que projeta) com idade de 22 anos, massa de $75 \mathrm{~kg}$, estatura de $1.75 \mathrm{~m}$ e com tempo de prática de 19 anos, e o uke (judoca que cai) com idade de 22 anos, massa de $72 \mathrm{~kg}$, estatura de $1.75 \mathrm{~m}$ e com tempo de prática de 15 anos.

Por ser um trabalho exploratório, é justificada a utilização de apenas dois sujeitos, com características físicas e técnicas mais próximas possíveis, além da dificuldade da coleta em função da disponibilidade de apenas um acelerô- metro, fixado em três locais diferentes do corpo para as coletas.

Para medir a aceleração $\left(\mathrm{m} / \mathrm{s}^{2}\right)$ durante os impactos do judoca projetado no tatame foi utilizado um acelerômetro triaxial do Tipo 4321 da Brüel \& Kjaer, confeccionado de titânio, com dimensões de 28.6 × 28.6 × $17 \mathrm{~mm}$, com capacidade máxima de choque de $1000 \mathrm{~g}$ (aceleração da gravidade).

A partir do atendimento das exigências legais conforme o que dispõem as Resoluções 196 e 251, de 07/08/97 do Conselho Nacional da Saúde, bem como a assinatura dos atletas de um consentimento informado, de acordo com as exigências no Comitê de Ética em Pesquisa com Seres Humanos da UFSC, processo aprovado sob o no 017/07, os dados foram coletados da seguinte forma: o acelerômetro foi fixado no judoca que era projetado segundo a metodologia adotada por Santos (2003), sendo no punho sobre a articulação radioulnar distal (ver figura 1); no quadril, na porção superior da crista ilíaca (ver figura 2) e no tornozelo, dois centímetros proximais ao maléolo medial (ver figura 3 ).

A fixação do acelerômetro e dos cabos foi efetuada com fita elástica de tal modo que permitisse a execução das técnicas de forma natural e para que não houvesse oscilação dos mesmos.

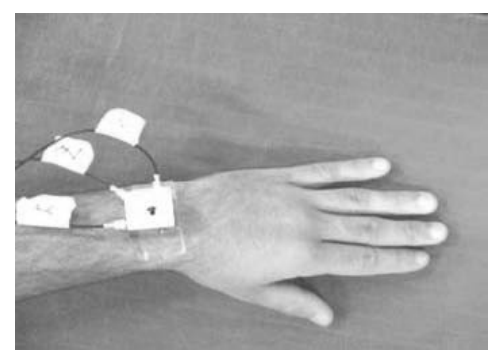

Figura 1. Fixação no punho 


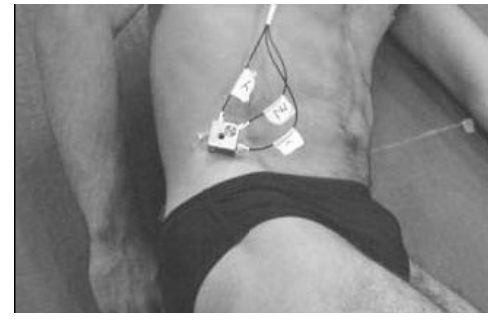

Figura 2. Fixação no quadril

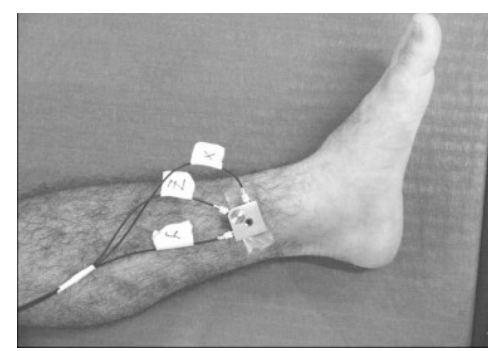

Figura 3. Fixação no tornozelo

Na posição anatômica, os eixos $\mathrm{x}, \mathrm{y}$ $\mathrm{e} z$ corresponderam respectivamente às direções ântero-posterior, vertical e látero-lateral (ver figura 4).

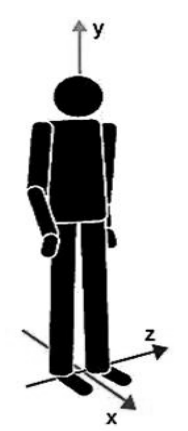

Figura 4. Direção de x, y e z na posição em pé

Porém, no momento do impacto, durante a realização do ukemi, o judoca cai no tatame na posição lateral, resultando nas direções de impacto $x, y$ e $z$ respectivamente látero-lateral, ânteroposterior e vertical (ver figura 5).

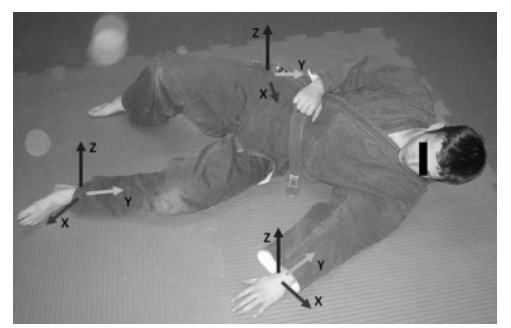

Figura 5. Direção de x, y e z na posição do ukemi
Para a coleta propriamente dita, montou-se uma área com 3 tatames sintéticos, compostos de copolímero etileno acetato de vinila (EVA), texturizado e siliconizado, cada um medindo $199.0 \times 99.0 \times 4.0 \mathrm{~cm}$ (ver figura 6), onde, após adaptação e aquecimento, os judocas realizaram 10 projeções e queda da técnica Ippon-Seoi-Nage para cada articulação corporal - punho, quadril e tornozelo.

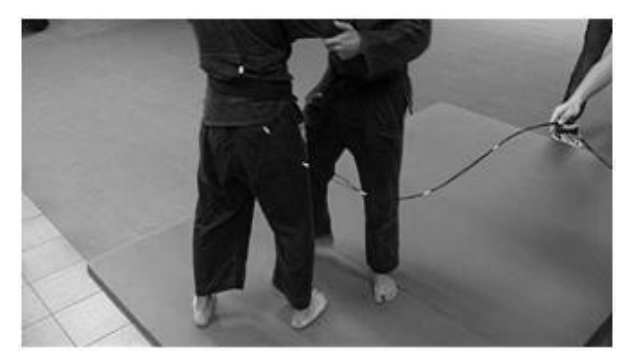

Figura 6. Área de 297.0 x $199.0 \mathrm{~cm}$ x $4.0 \mathrm{~cm}$, montada com três tatames para a execução das projeções

A escolha da técnica Ippon-Seoi-Nage para a realização do estudo é justificada pela: preferência pelos atletas investigados; pelas altas magnitudes de impacto que ela pode gerar no corpo do uke durante o ukemi, visto que o atleta é lançado de uma altura considerável em queda livre após a aplicação do golpe; e ainda, esta técnica é mostrada na literatura como sendo uma das mais executadas por judocas de categoria leve, além de ser um dos golpes mais frequentes que ocasionam lesões no judô (Barsottini, Guimarães, \& Morais, 2006).

As fases de execução da técnica Ippon-Seoi-Nage (ver figura 7), de acordo com Imamura et al. (2006), ocorrem da seguinte maneira: o kuzushi começa com o primeiro movimento do tori para a entrada do lançamento e termina com 
a colocação do pé de apoio do tori no chão de forma que ambos os pés estejam no chão. O tsukuri segue imediatamente o kuzushi e começa com tori empurrando os pés contra o chão e termina quando os calcanhares do uke começam a perder o contato com o chão. O kake segue o tsukuri e começa quando os dedos do pé do uke sobem do chão, sendo seu corpo lançado no ar, e termina quando qualquer parte de ambas as pernas toca no chão.

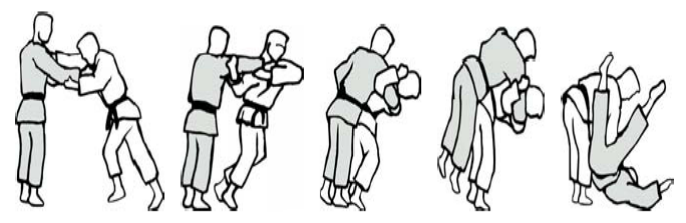

Figura 7. Fases de projeção da técnica Ippon-SeoiNage (adaptado de Imamura et al., 2006)

A carga elétrica gerada pelo transdutor piezoelétrico, durante a colisão do segmento corporal com o solo, nas três direções, passava por intermédio de 3 cabos (eixos $x, y$ e $z$ ) para seus respectivos pré-amplificadores para evitar saturação. A aquisição do sinal foi realizada pelo módulo MCS 1000 composto por 16 canais de até $\pm 10 \mathrm{~V}$, e depois processados pelo software de aquisição de sinais AqDados 7.02 e de análise AqDAnalysis 7. Para a retirada dos valores de impacto foi utilizado o valor de pico e o tempo de duração do impacto foi obtido pela subtração entre o tempo final e inicial da curva que caracterizava o impacto. Os sinais, depois de corrigidos por fatores de amplificação específicos para cada eixo, foram divididos por $9.81 \mathrm{~m} / \mathrm{s}^{2}$ para serem transformados em unidades de aceleração da gravidade (g).
Para a retirada dos valores de impacto foi utilizado o valor de pico, e para verificar os tempos de impacto de cada evento foi considerado o tempo inicial e final da curva que caracterizava a aterrissagem, conforme a figura 8 . O tempo de duração do impacto foi obtido pela subtração entre o tempo final e inicial.

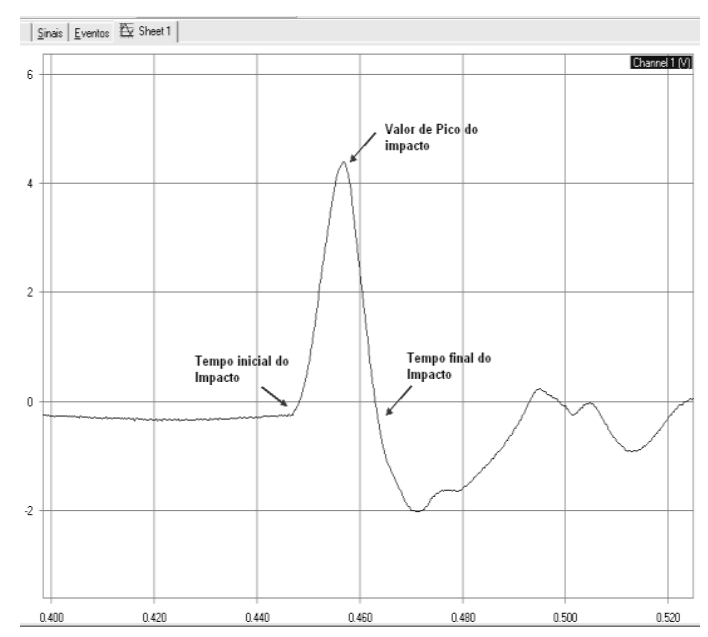

Figura 8. Critério para retirar o valor dos tempos de duração e o pico de impacto de cada evento

Os dados foram tratados estatisticamente por meio do programa SISVAR 5.0, utilizando a estatística descritiva (média, desvio padrão e coeficiente de variação) para a identificação das variáveis.

Para comparar os valores de impacto e os valores de tempo de duração dos impactos entre os eixos de movimento e entre as articulações corporais, inicialmente aplicou-se um teste de Shapiro-Wilk para comprovar a normalidade dos dados e posteriormente utilizou-se a análise de variância multifatorial (MANOVA three-way), com um intervalo de confiança de $95 \%$ a análise de variância a $p<.05$. 


\section{RESULTADOS}

As magnitudes dos impactos (g) nas coordenadas $\mathrm{x}, \mathrm{y}$ e $z$, medidos no punho, quadril e tornozelo durante $\mathrm{o}$ ukemi na técnica Ippon-Seoi-Nage estão dispostas na tabela 1 .
Observando a tabela 1 verificam-se altos valores de impactos tanto no punho quanto no tornozelo do judoca, assim como a heterogeneidade dos valores em todas as articulações avaliadas.

Tabela 1

Média $(M)$, desvio padrão $(D P)$, valores máximos e mínimos e coeficiente de variação $(C V)$ das magnitudes dos impactos (g) medidos durante o ukemi na técnica Ippon-Seoi-Nage no punho, quadril e tornozelo, no eixo látero-lateral (x), ântero-posterior $(y)$ e vertical $(z)$

\begin{tabular}{lccccccccc}
\hline Local & \multicolumn{3}{c}{ Punho } & \multicolumn{3}{c}{ Quadril } & \multicolumn{3}{c}{ Tornozelo } \\
\cline { 2 - 10 } Eixos & $\mathrm{x}$ & $\mathrm{y}$ & $\mathrm{z}$ & $\mathrm{x}$ & $\mathrm{y}$ & $\mathrm{z}$ & $\mathrm{x}$ & $\mathrm{y}$ & $\mathrm{z}$ \\
\hline$M(\mathrm{~g})$ & 140.21 & 75.99 & 351.95 & 9.33 & 5.18 & 13.51 & 76.09 & 101.97 & 242.89 \\
DP (g) & 40.24 & 43.36 & 96.59 & 4.15 & 1.54 & 5.44 & 55.06 & 53.52 & 142.01 \\
Máx. (g) & 218.55 & 128.24 & 447.81 & 17.45 & 7.84 & 24.60 & 183.51 & 169.52 & 539.67 \\
Mín. (g) & 73.39 & 17.64 & 140.57 & 4.58 & 3.48 & 5.50 & 27.28 & 29.58 & 117.92 \\
CV (\%) & 28.70 & 57.06 & 27.44 & 44.46 & 29.78 & 40.27 & 72.36 & 52.48 & 58.47 \\
\hline
\end{tabular}

Os valores dos tempos de impactos (segundos) nas coordenadas $\mathrm{x}, \mathrm{y} \mathrm{e} z$, medidos no punho, quadril e tornozelo durante a técnica Ippon-Seoi-Nage estão dispostos na tabela 2 .

Tabela 2

Média (M), desvio padrão (DP), valores máximos e mínimos e coeficiente de variação $(C V)$ dos tempos dos impactos (s) medidos durante a técnica Ippon-Seoi-Nage no punho, quadril e tornozelo, no eixo látero-lateral ( $x$ ), antero-posterior $(y)$ evertical $(z)$

\begin{tabular}{lccccccccc}
\hline Local & \multicolumn{3}{c}{ Punho } & \multicolumn{3}{c}{ Quadril } & \multicolumn{3}{c}{ Tornozelo } \\
\cline { 2 - 10 } Eixos & $\mathrm{x}$ & $\mathrm{y}$ & $\mathrm{z}$ & $\mathrm{x}$ & $\mathrm{y}$ & $\mathrm{z}$ & $\mathrm{x}$ & $\mathrm{y}$ & $\mathrm{z}$ \\
\hline$M(\mathrm{~g})$ & .010 & .005 & .011 & .042 & .026 & .587 & .006 & .011 & .014 \\
$D P(\mathrm{~g})$ & .002 & .002 & .003 & .010 & .011 & .098 & .003 & .010 & .003 \\
Máx. (g) & .014 & .012 & .016 & .059 & .043 & .687 & .012 & .040 & .018 \\
Mín. (g) & .006 & .004 & .008 & .026 & .011 & .354 & .003 & .006 & .010 \\
CV (\%) & 23.93 & 46.26 & 23.07 & 23.33 & 44.24 & 16.73 & 44.37 & 91.12 & 17.69 \\
\hline
\end{tabular}

Os valores dos tempos de impactos apresentados nas articulações, com excepção do quadril no eixo $z$, são todos de curta duração $(<.01 \mathrm{~s})$, de acordo com o critério de Macaulay (1987).
Os resultados das comparações das magnitudes e tempos de duração de impacto entre os eixos ( $\mathrm{x}, \mathrm{y}, \mathrm{z})$ e entre as articulações corporais (punho, quadril e tornozelo) estão dispostos na tabela 3 . 
Tabela 3

Comparação das magnitudes ( $g$ ) e tempos de duração dos impactos (s) entre os eixos ( $x, y$ e $z$ ), e entre as articulações (punho, quadril e tornozelo)

\begin{tabular}{ccccc}
\hline Local & Variável & $\mathrm{x}$ & $\mathrm{y}$ & $\mathrm{z}$ \\
\hline \multirow{2}{*}{ Punho } & Impacto & 140.21 & 75.99 & $351.95^{* *}$ \\
& Tempo & .010 & .004 & .011 \\
Quadril & Impacto & $9.33^{*}$ & 5.18 & $14.91^{*}$ \\
& Tempo & .043 & .025 & $.650^{* * *}$ \\
Tornozelo & Impacto & 76.09 & $101.97^{*}$ & $242.89^{* * *}$ \\
& Tempo & .070 & .019 & .014 \\
\hline Nota: ${ }^{*}$ diferenças significativas entre os eixos, $p<.05 ; * *$ diferenças significativas entre as articulações \\
corporais, $p<.05$
\end{tabular}

$\mathrm{Na}$ comparação entre os eixos, observa-se na tabela 3 que no punho as magnitudes de impacto no eixo $z$ foram significantemente maiores que os eixos $\mathrm{x}$ e $\mathrm{y}$. No quadril, somente os tempos de duração dos impactos foram maior no eixo $z$, e no tornozelo, as magnitudes de impacto no eixo $z$ foi significativamente maiores que os eixos $\mathrm{x}$ e $\mathrm{y}$.

$\mathrm{Na}$ comparação entre as articulações, as magnitudes dos impactos no quadril, no eixo $x$ e $z$, foram significativamente menores do que nas demais articulações, e no tornozelo, os impactos no eixo y e $z$ foram maiores. Os tempos de duração dos impactos foram maiores no quadril, no eixo $z$.

\section{DISCUSSÃO}

A grande variabilidade dos dados observada no tornozelo (tabelas 1 e 2) pode ter ocorrido devido a dificuldade no controle do movimento do quadril e membros inferiores ao longo da execução do ukemi. De acordo com Santos e Melo (2001), durante a queda, o atleta realiza uma sequência de movimentos com mudanças sucessivas do eixo de rotação do corpo, até o momento da colisão, que inicia com a batida controlada da mão no tatame, seguido do apoio do ombro que serve como eixo de rotação para o quadril, que, por conseguinte, forma um novo eixo para rotação dos membros inferiores.

Outro fator que também pode ter influenciado nos altos valores do $\mathrm{CV}$ das magnitudes de impactos é a caracterização das projeções como uma tarefa motora aberta, cuja execução sofre interferência da reação do uke. A situação laboratorial pode ter interferido em função da limitação da movimentação dos atletas, visto que os cabos do acelerômetro eram fixados no corpo. O tempo estipulado entre uma projeção e outra (30s para prevenir estresse) pode ter feito com que o tori mantivesse o ritmo de execução, e assim, alterando os valores de impacto. Em situação real, tanto de treinamento quanto de competição, as magnitudes dos impactos provavelmente seriam muito maiores.

Com relação às magnitudes de impacto nos diferentes eixos de movimento (tabela 3 ), verificou-se que os valores médios de impactos obtidos no eixo $z$ (vertical) foram maiores que os demais, seguidos pelos valores do eixo x (látero-lateral) e em menores valores no eixo y (longitudinal). Este fato ocor- 
re devido a técnica de amortecimento de queda chamada Zempô-Kaiten-Ukemi utilizada pelo judoca quando é projetado por este tipo de técnica (Ippon-SeoiNage), na qual ele gira sobre os ombros e bate primeiramente com a mão no tatame, nas direções vertical e médiolateral, justificando os valores gradativos de $z$ para x e para y. A ação da força gravitacional e da velocidade com que o atleta é projetado também contribui para o aumento das forças de impacto no sentido vertical no momento da colisão.

Quanto à comparação das magnitudes de impacto entre as articulações corporais, verifica-se que os maiores valores foram registados no punho e no tornozelo, ambos no sentido vertical. Esses resultados são justificados pelo fato da mão ser o primeiro segmento a tocar o solo. Segundo Santos (2003) esse gesto faz com que ocorra uma transferência da quantidade de movimento da mão através do braço para as demais partes do corpo. Já o tornozelo percorre uma trajetória maior, devido ao comprimento do raio, formado pela distância entre o quadril e o pé, gerando uma grande velocidade tangencial, e consequentemente, um grande valor de impacto (Santos, 2003). Outro fator que pode ter influenciado nas maiores magnitudes de impacto no punho e no tornozelo é a massa relativamente pequena desses segmentos. A massa corporal proporciona variações no tempo de contato entre os corpos, ou seja, quanto maior a massa, maior é a deformação dos corpos e maior é o tempo de contato entre eles, o que diminui as magnitudes da força de impacto gerada (Nussenzveig, 1996). Por isso, para que se possa inferir cor- retamente na relação entre massa e forças impactantes, torna-se importantíssimo considerar ainda a atuação de elementos dissipativos que atuam no momento da colisão, como a deformação dos tecidos corporais e do piso ou tatame.

Já na região do quadril foram registadas as menores magnitudes de impacto em relação aos outros locais investigados. Os resultados foram similares aos encontrados por Santos (2003), que investigou os valores das magnitudes de impactos em judocas sendo projetados também pela técnica Ippon-Seoi-Nage, em diferentes tipos de tatames, e encontrou valores de impactos no quadril menores do que os no tornozelo e no punho. O principal fator que ameniza os valores de impacto gerados no quadril é a antecipação da batida controlada da mão no tatame, seguido do apoio do ombro que serve como eixo de rotação para o quadril durante o rolamento do $u k e$, dissipando grande parte do impacto advindo da colisão do quadril do judoca com o tatame. A importância dessa dissipação, mesmo que ainda parecendo pouca, é que, segundo Mertz (1993), o risco de lesões na pélvis é mais alto do que em outras partes do corpo, devido a sua localização, e principalmente porque essa região possui maior massa e maior número de órgãos internos que seriam facilmente lesionados durante fortes impactos.

Quanto à comparação dos valores de tempo de impacto entre os eixos, o eixo vertical ( $z$ ) foi o de maior duração. O critério de Macaulay (1987) aponta uma tendência global de que, quanto menor a duração do evento, maiores acelerações o organismo suporta. Con- 
siderando que o eixo vertical foi o que obteve maiores magnitudes e maiores tempos de duração dos impactos, podese deduzir que nesta direção ocorre a maioria das lesões advindas dos impactos no judô. Santos e Melo (2003) concluíram em um estudo descritivo longitudinal que, grande parte das lesões sofridas por judocas era proveniente das quedas realizadas de forma errada. Cair de forma errada significa não realizar a técnica de amortecimento correta, não ocorrendo distribuição das forças de impacto. Esta prática é comum, visto que um dos critérios para a pontuação no judô é a forma como o judoca cai. Portanto, não cair bem significa menor pontuação para o adversário, o que em muitos casos acabam gerando lesões, principalmente na região glenoumeral do uke (Santos \& Melo, 2003).

Com relação aos tempos de duração dos impactos entre as articulações analisadas, os menores valores foram registrados no punho. Esse fato ocorre devido à grande velocidade que a mão atinge o solo, empregando uma grande velocidade de separação entre o corpo do judoca e o tatame e, consequentemente, um menor tempo de duração do impacto. Observa-se ainda que essa região sofreu as maiores magnitudes de impacto, pois o valor do impacto (desaceleração) das partes envolvidas na batida (corpo do judoca e tatame) é inversamente proporcional ao tempo de duração do impacto. Já os maiores tempos de duração dos impactos foram registrados no quadril, sendo esses resultados também encontrados por Santos (2003). Esses resultados mostram que, pelo fato do quadril estar localizado próximo do centro de massa corporal, este possui maior massa, o que aumenta a deformação do tatame durante a colisão, e consequentemente, o tempo de contato.

Referenciando os dados encontrados com o critério de Macaulay (1987) para a tolerância do corpo inteiro as vibrações, pode-se afirmar que os valores médios das magnitudes de impacto no punho (351.95 no eixo z) e tornozelo (242.89 no eixo $z$ ), medidas na técnica Ippon-Seoi-Nage, ultrapassam o limite de impacto citado para que não haja severas lesões (200 g). Porém, os tempos de duração desses eventos (< 10 - 1) podem ser considerados como de curta duração, portanto não causadores de lesões. Quanto aos ao tempo de impacto na região do quadril, registado no eixo vertical, os valores chegam a ser maior que $1 / 2$ segundo, o que representa um pulso de longa duração. Porém, de acordo com o referido critério, as magnitudes de impacto geradas nessa região (menores que $14 \mathrm{~g}$ ), não são suficientes para que ocorram lesões.

Como já apontado anteriormente, o critério de Macaulay (1987) não limita o tempo total diário de exposição permitido, como cita as ISO 5349 (1986) e ISO 2631 (1985) para eventos periódicos, como também não aponta o número de repetições e nem intervalo de descanso entre os impactos. Sem o controle destas variáveis não se pode fazer qualquer inferência sobre a capa cidade de auto-reparo dos biomateriais frente às características das cargas e ao processo de fadiga, advindo das quedas no judô. Por outro lado, de acordo com Radin, Paul e Rose (1998), partindo do pressuposto de que, quanto menores forem as durações dos impactos, maiores são as magnitudes que o corpo pode 
sustentar, os impactos por si só não acometeriam danos ao organismo, porém, a repetitividade destes sim. Podem-se salientar assim os possíveis efeitos deletérios ao corpo do judoca ao longo do tempo de prática, considerando o alto número de quedas realizadas em competições e principalmente em treinos, que podem chegar a uma média de $21.46 \pm 8.46$ quedas por sessão de treino para atletas amadores (Melo, Santos, Teixeira, \& Cunha, 2005).

Ainda, para que se possa avaliar precisamente o efeito dos impactos no organismo de judocas, estudos experimentais devem ser realizados controlando variáveis como: o tipo de tatame utilizado, a velocidade de execução do golpe, a massa corporal do uke, a realização correta do ukemi o número de quedas por treino e o tempo de descanso entre as quedas.

Alguns fatores que interferem no número e na qualidade de execução do ukemi são o tipo e a fase de treinamento dos judocas, bem como o nível técnico do tori, pois uma projeção bem realizada, dando segurança ao uke, é imprescindível para a realização de um ukemi que dissipe a energia contida no impacto mecânico no tatame, protegendo o organismo do judoca.

\section{CONCLUSÕES}

Considerando os resultados obtidos, o referencial teórico e as limitações do estudo, foi possível observar que: a) as maiores magnitudes dos impactos foram encontradas no eixo vertical, em todas as regiões corporais analisadas; b) o quadril apresentou as menores magnitudes de impactos em todos os eixos, e o punho foi maior que o tornozelo apenas no eixo láterolateral; c) o maior tempo de duração dos impactos ocorreu no eixo vertical, em todas as regiões corporais investigadas; e, d) o quadril foi o local que apresentou maior tempo de duração de impacto.

Por fim, podemos concluir que é de grande importância a realização de outras investigações mais aprofundadas sobre o tema, visto que as características dos impactos mecânicos (magnitudes e tempo de duração) obtidas neste estudo podem gerar efeitos deletérios ao organismo dos judocas ao longo de anos de prática. Vale também evidenciar aos sensei (professores) e judocas a importância da prática correta e constante do ukemi, tanto para os judocas iniciantes, quanto para os experientes. Torna-se necessário desenvolver metodologias de prática que enfatizem sempre a correta execução de cada tipo de ukemi, específico para cada tipo de projeção, respeitando durante os treinos os intervalos entre projeções e a segurança da queda, pregada pela "máxima" do judô Jita-Kyoei (prosperidade e benefícios mútuos). Com tais medidas é possível minimizar o processo de fadiga do biomaterial e de sobrecarga ao qual o organismo é submetido constantemente durante o treinamento.

\section{REFERÊNCIAS}

Barrault, D., Achou, B., \& Sorel, R. (1983). Accidents et incidents survenus au cours des compétitions de judo. Symbian, 15, 144-152.

Barsottini, D., Guimarães, A. E., \& Morais, P. R. (2006). Relação entre técnicas e lesões em praticantes de judô. Revista Brasileira de Medicina do Esporte, 12(1), 56-60. 
Boyer, K. A., \& Nigg, B. M. (2004). Muscle activity in the leg is tuned in response to impact force characteristics. Journal of Biomechanics, 37, 1583-1588.

British Standards Institution (1987). Guide to the evaluation of human exposure to whole-body mechanical vibration and repeated shock. Londres: Autor.

Derrick, T. R., \& Mercer, J. A. (2004). Ground/foot impacts: Measurement, attenuation, and consequences. Medicine $\mathcal{E}$ Science in Sports \& Exercises, 34(6), 830-831.

Esteves, A. C., Nascimento, A. S., Moreira, F. D., \& Reis, D. C. (2007). Impacto no eixo ântero-posterior no chute Bandal Tchagui do Taekwondo. Lecturas: Educación Física y Deporte, 104. Acedido em Maio, 20, 2009 a partir de http://www.efdeportes.com/efd104/ta ekwondo.htm

Fraga, L. A. C. (2002). Presença de atitudes escolióticas em meninos judocas e não judocas. Dissertação de Mestrado, Ciências do Movimento Humano, Escola de Educação Física, Universidade Federal do Rio Grande do Sul, Rio Grande do Sul, Brasil.

Gottschall, J. S., \& Kram, R. (2005). Ground reaction forces during downhill and uphill running. Journal of Biomechanics, 38, 445-452.

Griffin, M. J. (1990). Handbook of human vibration, London: Academic Press.

Harris, C., \& Piersol, A. (2002). Shock and vibration handbook ( $5^{\text {a }}$ edição).New York: McGraw-Hill.

Hatze, H. (1992). The effectiveness of grip bands in reducing racquet vibration transfer and slipping. Medicine $\mathcal{E}$ Science in Sports \& Exercise, 24, 226-230.

Hennig, E. M., Rosenbaum, D., \& Milani, T. L. (1992) Transfer of tennis racket vibrations onto the human forearm. Medicine \& Science in Sports \& Exercise, 24, 1134-1140.
Imamura, R. T., Hreljac, A., Escamilla, R. F., \& Edwards, W. B. (2006). A threedimensional analysis of the center of mass for three different judo throwing techniques. Journal of Sports Science and Medicine, 5, 122-131.

International Standard (1985). Evaluation of human exposure to whole-body vibration: Part 1 - General requirements. ISO 2631 1985.

International Standard (1986). Mechanical vibration: Guidelines for the measurement end the assessment of human exposure to hand-transmitted vibration. ISO 5349 1986.

Ishitake, T., Kano, M., Miyazaki, Y., Ando, H., Tsutsumi, A., \& Matoba, T. (1998). Whole-body vibration suppresses gastric motility in healthy men. Industrial Health, 36(2), 93-97.

James, G., \& Pieter, W. (2003). Injury rates in adult elite judoka. Biology of Sport, 20(1), 25-32.

Jarrah, M., Qassem, W., Othman, M., \& Gdeisat, M. (1997). Human body model response to mechanical impulse. Medical Engineering \& Physics, 19(4), 308-316

Lafortune, M. A., Henning, E., \& Valiant, G. A. (1995). Tibial shock measured with bone and skin mounted transducers. Journal of Biomechanics, 28(8), 989993.

Leavitt, F. J. (2003). Can martial arts falling techniques prevent injuries? Injury Prevention, 9, 284.

Levy, M., \& Smith, G. A. (2005). Effectiveness of vibration damping with bicycle suspension systems. Sports Engineering, 8, 99-106.

Macaulay, M. (1987). Introduction to impact engineering. London: Chapman and Hall.

Melo, S. I. L., Santos, S. G., Teixeira, J. S., \& Cunha, A. G. (2005). Estudo da distribuição dos impactos mecânicos em sessões de treinamento de judô. Revista 
Brasileira de Atividade Física e Saúde, 10(1), 76.

Mertz, J. H. (1993). Anthropomorphic test devices. In A. M. Nahum \& J. W. Melvin (Eds.), Accidental injury: Biomechanics and prevention (pp. 66-84). New York: Springer.

Nussenzveig, H. M. (1996). Curso de física básica 1: Mecânica (3 $3^{a}$ edição). São Paulo: Editora Edgard Blucher.

Orendurff, M. (1997). The effects of mountain bicycle fork stiffness on impact acceleration. Acedido em Julho, 9, 2008 a partir de http://www.cev.org./biblioteca/

Pieter, W., Talbot, C., Pinlac, V., \& Bercades, L. T. (2001). Injuries at the Konica Asian Judo championships. Acta Kineseologiae Universitatis Tartuensis, 6, 102-111.

Piucco, T. (2007). Análise dos impactos gerados durante a queda em três técnicas do Judô. Monografia, Centro de Desportos, Universidade Federal de Santa Catarina, Santa Catarina, Brasil.

Piucco, T., Santos, S.G., Pacheco, A. G., Souza, P. D., \& Piucco, D. C. (2007, Maio). Magnitude dos impactos durante as aterrissagens no basquete associado com lesões nos membros inferiores. Comunicação apresentada no XII Congresso Brasileiro de Biomecânica, Rio Claro, Brasil.

Radin, E. L., Paul, I. L., \& Rose, R. M. (1998). Role of mechanics factors in pathogenesis of primary osteoarthritis. The Lancet, 1, 519-521.

Santos, S. G. (2003). Estudo das características de impacto e da percepção humana de conforto na prática de ukemis em diferentes tatames. Tese de Doutoramento, Departamento de Engenharia de Produção, Universidade Federal de Santa Catarina, Santa Catarina, Brasil.

Santos, S. G. \& Melo, S. I. L. (2001). Biomecânica aplicada ao judô. In E. Franchini (Ed.), Judô: Desempenho competitivo (pp. 97-125). São Paulo: Manole.
Santos, S. G., \& Melo, S. I. L. (2003). Os "ukemis" e o judoca: Significado, importância, gosto e desconforto. Revista Brasileira de Cineantropometria e Desempenho Humano, 5(2), 33-43.

Santos, S. G., \& Shigunov, V. (2001). Percepção do atleta sobre as causas de suas lesões. FIEP Bulletin, 16, 273-276.

Shmitt, K. U., Niederer, P., \& Walz, F. (2004). Trauma biomechanics: Introduction to accidental injury. New York: Springer.

Sterkowicz, S. (1999). Body injury of youth training in Judo. JudoInfo Research. Acedido em Janeiro, 3, 2008 a partir de http://www.judoinfo.com/research4.ht $\mathrm{m}$

Voloshin, A. S. (2004). Propagação do impacto e seus efeitos sobre o corpo humano. In V. Zatsiorsky (Ed), Biomecânica no esporte: Performance e prevenção da lesão (pp. 452-459). Rio de Janeiro: Guanabara Koogan. 